\title{
Partial cooperation in strategic multi-sided decision situations
}

\author{
Subhadip Chakrabarti ${ }^{1} \cdot$ Robert P. Gilles $^{1} \cdot$ Emiliya Lazarova $^{2}(\mathbb{D}$
}

Published online: 26 June 2018

(c) The Author(s) 2018

\begin{abstract}
We consider a normal-form game in which there is a single exogenously given coalition of cooperating players that can write a binding agreement on pre-selected actions. The actions representing other dimensions of the strategy space remain under the sovereign, individual control of the players. We consider a standard extension of the Nash equilibrium concept denoted as a partial cooperative equilibrium as well as an equilibrium concept in which the coalition of cooperators has a leadership position. Existence results are stated and we identify conditions under which the various equilibrium concepts are equivalent. We apply this framework to existing models of multi-market oligopolies and international pollution abatement. In a multi-market oligopoly, typically, a merger paradox emerges in the partial cooperative equilibrium. The paradox vanishes if the cartel attains a leadership position. For international pollution abatement treaties, cooperation by a sufficiently large group of countries results in a Pareto improvement over the standard tragedy of the commons outcome described by the Nash equilibrium.
\end{abstract}

Keywords Non-cooperative game Partial cooperation $\cdot$ Partial cooperative equilibrium $\cdot$ Leadership equilibrium $\cdot$ Multi-market oligopoly $\cdot$ International pollution abatement

We would like to thank two referees of this journal and Lina Mallozzi for her valuable comments on the previous drafts of this paper. We also thank seminar participants at Queen's University Belfast; 9th Conference of the Society of Economic Design, Istanbul, Turkey, 2015; ASSET Meetings, Aix en Provence, France, 2014; NUI Galway Departmental Seminar, Galway, Ireland, 2013; UECE Lisbon Meetings, Lisbon, Portugal, 2012; and the Association of Public Economic Theory Annual Conference, Istanbul, Turkey, 2010..

$凶 \quad$ Emiliya Lazarova

E.Lazarova@uea.ac.uk

1 Queen's University Management School, Queen's University Belfast, Riddel Hall, 185 Stranmillis Road, Belfast BT9 5EE, UK

2 School of Economics, University of East Anglia, Norwich Research Park, Norwich NR4 7TJ, UK 


\section{Introduction}

We consider a game theoretic framework for understanding collaborative decision situations embedded in competitive environments. Often, these kinds of agreements on a specific economic issue involve a single group of decision makers who cooperate. This coalition writes a binding agreement concerning certain exogenously given jointly decided actions, while the non-collaborative decision makers remain uninvolved. Moreover, while decision makers subject to this binding agreement may cooperate on certain issues, these same players may act non-cooperatively when interacting with all other players along other dimensions. Examples where these tools are applicable range from areas as diverse as environmental agreements (Barrett 1990; Carraro and Siniscalco 1993), R\&D collaborations (Yi and Shin 2000; Banal-Español et al. 2013), and financial alliances between banks (White 1996; Popov and Ongena 2011; In't Veld and van Lelyveld 2014).

A rigorous analysis of these games with partial cooperation calls for the development of specifically tailored equilibrium concepts. Our notion of partial cooperative equilibrium extends standard Nash best response rationality to our framework and is similar to the coalition equilibrium concept of Ichiishi (1981). Here, the cooperating players write a binding agreement with regard to a specific set of actions, and, simultaneously, act non-cooperatively with regard to their individualistic or "private" strategy. The non-cooperators act independently from the cooperators and select a standard best response strategy to all other players' actions. By supplementing the strategy space of the cooperators with a private strategy, this definition generalises the concept of a partial cooperative game in Chakrabarti et al. (2011). Our partial cooperative equilibrium existence result extends the existence theorems seminally stated in Mallozzi and Tijs (2008a, 2009, 2012) for more restricted environments. Our result is based on techniques seminally developed by Glicksberg (1952).

Next, we consider a leadership equilibrium concept, which postulates that the cooperating coalition has a (Stackelberg) leadership position. Hence, after a binding agreement has been signed by the cooperative players, all players-cooperators as well as non-cooperators - make independent decisions with regard to all other actions.

The underlying sequential decision process in a normal-form game was seminally discussed and developed in Section 3.5 of Ray (2007). This structure was first implemented to partial cooperative games by Mallozzi and Tijs (2008a) and subsequently extended in Mallozzi and Tijs (2008b, 2012) and Chakrabarti et al. (2011). Our leadership equilibrium notion builds on this work. Mallozzi and Tijs (2008a) proposed this concept for the class of symmetric potential games. In a subsequent study, Mallozzi and Tijs (2009) consider symmetric aggregative games. The situation when the cooperating agents are faced with multiple Nash equilibria when interacting with the non-cooperative agents is discussed in Mallozzi and Tijs (2008b). Chakrabarti et al. (2011) extended this further to arbitrary non-cooperative games.

We design an extension to the leadership equilibrium notion along several dimensions. First, we extend the strategy space for the cooperators, such that actions not subject to coalitional decision-making are decided simultaneously by all playerscooperators as well as non-cooperators. Second, we consider a generalised aggregation of the payoffs of the cooperators to evaluate coalitional decisions. A commonly applied 
aggregator is the utilitarian aggregator. The utilitarian aggregator is imposed, for example, in the theory of (standard) partial cooperative games as seminally developed in Mallozzi and Tijs (2009). Alternative aggregators that can be handled by our generalised framework are the Rawlsian aggregator (Rawls 1999) and the Nashian aggregator (Nash 1950). Third, following Chakrabarti et al. (2011), we assume that in the case of multiple Nash equilibria, the cooperators act optimistically and employ a max-max strategy with regard to their payoffs. Finally, we study the existence properties of our notions in a much larger class of normal-form games.

We investigate two applications of our general framework. In the first application, we borrow the model of cartel formation in a multi-market Cournot oligopoly from Billand et al. (2014). Firms compete in two separate markets. Cooperators collude with regard to quantity choice in one market and not in the other. In the partial cooperative equilibrium, cooperators are worse off than in the standard competitive Cournot-Nash equilibrium due to the merger paradox (Salant et al. 1983) if the market on which cooperation occurs is sufficiently large relative to the competitive market. If the market on which firms cooperate is sufficiently small, however, it may be the case that partial cooperation leads to strictly higher payoffs to all firms compared to the standard competitive equilibrium. On the other hand, in the leadership equilibrium, cartel members as well as regular competitors are better-off relative to the Nash equilibrium, achieving a strict Pareto improvement, thereby restoring a clear incentive to establish a cartel in such a multi-market oligopoly.

In the second application, we consider the effects of international pollution abatement treaties. Such treaties are best described as partial cooperative agreements: A single coalition of treaty countries writes a binding agreement on certain aspects of the spectrum of economic controls at the disposal of a country's government. The 1997 Kyoto treaty, for example, only regulated emissions of carbon dioxide ("carbon production") rather than the carbon consumption, which normally results from many other economic variables (Helm 2012; Newell et al. 2013).

Our partial cooperative approach supplements Chander and Tulkens (1997)'s model of emissions control with an additional labour input besides the usual polluting factor. Cooperators choose the amount of labour freely, but form a cooperative agreement with regard to the amount of pollutants. In this context, there is no difference between the partial cooperative equilibrium and the leadership one, but both differ from the Nash outcome. Here, only cooperators reduce the amount of pollutants and the level of reduction is greater, the larger is the number of cooperators. All non-treaty countries act as free riders in these equilibrium situations.

\section{Generalised partial cooperative games}

We consider normal-form games in which an ex-ante postulated group of players collaborates and writes binding agreements on a given subset of actions. All players outside this coalition of cooperators are assumed to follow their individual objectives.

Throughout, let $C=\{1, \ldots, k\}$ be a given and pre-determined coalition of cooperators, where $k \geqslant 2$. Furthermore, let $N=\{1, \ldots, n\}$ with $n \geqslant 1$ be the set of non-cooperative players. Thus, the population of all players is given by $C \cup N$, con- 
sisting of $k$ cooperating players and $n$ individualistic players. We indicate a generic member of the coalition of cooperators by $i \in C$, while a generic non-cooperative player is denoted by $j \in N$. We consider three separate types of actions:

- Each individual $i \in C$ controls the selection of a private action $x_{i} \in X_{i}$, where $X_{i} \neq \varnothing$ denotes $i$ 's private action set. We let $X=\prod_{i \in C} X_{i}$ be the private action tuple set of the coalition of cooperators $C$.

- The coalition of cooperators $C$ selects cooperatively a collective action $y \in Y \neq$ $\varnothing .^{1}$

- Finally, each non-cooperator $j \in N$ selects an individual action $z_{j} \in Z_{j} \neq \varnothing$. We denote $Z=\prod_{j \in N} Z_{j}$ the non-empty action tuple set of the group of noncooperators $N$.

Given the three types of actions, we now denote by

$$
a=(x, y, z) \in A \equiv X \times Y \times Z
$$

a general action tuple, where $A$ is the space of all feasible action tuples.

In addition, following accepted conventions, for every cooperator $i \in C$ and private action tuple $x \in X$, we denote by $x_{-i}=\left(x_{1}, \ldots, x_{i-1}, x_{i+1}, \ldots, x_{k}\right)$ the actions assigned to all other cooperators in $C$. Similarly, for every non-cooperator $j \in N$ and action tuple $z \in Z$, we denote by $z_{-j}=\left(z_{1}, \ldots, z_{j-1}, z_{j+1}, \ldots, z_{n}\right)$ the actions assigned to all other non-cooperators in $N$.

Moreover, each cooperating player $i \in C$ is endowed with a payoff function $u_{i}: A \rightarrow \mathbb{R}$ and non-cooperator $j \in N$ is similarly endowed with a payoff function $v_{j}: A \rightarrow \mathbb{R}$. We denote by $u=\left(u_{1}, \ldots, u_{k}\right)$ the list of payoff functions over all cooperators, and by $v=\left(v_{1}, \ldots, v_{n}\right)$ the list of payoff functions for all noncooperators.

The collective decisions of the coalition of cooperators $C$ are guided by some aggregation of the payoffs of its members (Negishi 1963). Formally, we introduce an aggregator as a function $\Lambda: \mathbb{R}^{k} \rightarrow \mathbb{R}$, such that the coalition of cooperators $C$ evaluates each action tuple $a=(x, y, z) \in A=X \times Y \times Z$ through the aggregated payoff function $U: A \rightarrow \mathbb{R}$ defined by the following:

$$
U(a)=U(x, y, z)=\Lambda\left(u_{1}(x, y, z), \ldots, u_{k}(x, y, z)\right) .
$$

We say that the aggregator $\Lambda$ is Paretian if it is non-decreasing in $u_{i}, i \in C$. Throughout, we refer to two common Paretian aggregators. The Rawlsian aggregator is given by $\Lambda^{r}\left(u_{1}, \ldots, u_{k}\right)=\min \left\{u_{1}, \ldots, u_{k}\right\}$, while the utilitarian aggregator is defined as $\Lambda^{u}\left(u_{1}, \ldots, u_{k}\right)=\sum_{i=1}^{k} u_{i}$

Clearly, the collective payoff function $U$ is founded on the aggregator $\Lambda$ and the payoff functions $u_{1}, \ldots, u_{k}$ of its members.

\footnotetext{
1 This generalises Mallozzi and Tijs (2008a) and Chakrabarti et al. (2011), in which the set of collective actions, $Y$, is modelled as a $k$-dimensional vector space with $Y=\prod_{i \in C} Y_{i}$. In this case, $y_{i} \in Y_{i}$ denotes the individual player's $i \in C$ strategic (sub-)action as part of the collective action $y \in Y$. In many applications, it is additionally assumed that, for every cooperator $i \in C$, the action set $Y_{i}$ is, in fact, some subset of a Euclidean space.
} 
Definition 2.1 A generalised partial cooperative game is a list $\Gamma=\langle C, N, X, Y, Z$, $u, v, \Lambda\rangle$.

Note, here as well, that the case where $Y$ is a singleton set reverts any generalised partial cooperative game to a standard $(k+n)$-player normal-form game.

\subsection{Partial cooperative equilibrium}

This equilibrium notion extends Nash's best response rationality to all decisions, namely the individual and private actions of the players as well as the collective action of the coalition of cooperators. The latter evaluates the outcomes of their selection through the aggregated payoff function $U$ based on the aggregator $\Lambda$.

Definition 2.2 An action tuple $a^{*}=\left(x^{*}, y^{*}, z^{*}\right) \in A$ is called a partial cooperative equilibrium in $\Gamma=\langle C, N, X, Y, Z, u, v, \Lambda\rangle$ if it satisfies the following conditions:

(i) For every cooperating player $i \in C$, it holds that

$$
u_{i}\left(x^{*}, y^{*}, z^{*}\right) \geqslant u_{i}\left(x_{i}, x_{-i}^{*}, y^{*}, z^{*}\right)
$$

for every private action $x_{i} \in X_{i}$.

(ii) For the coalition of cooperators $C$, it holds that

$$
U\left(x^{*}, y^{*}, z^{*}\right) \geqslant U\left(x^{*}, y, z^{*}\right)
$$

for every collective action $y \in Y$, where $U$ is given by (2).

(iii) In addition, for every non-cooperator $j \in N$, it holds that

$$
v_{j}\left(x^{*}, y^{*}, z^{*}\right) \geqslant v_{j}\left(x^{*}, y^{*}, z_{j}, z_{-j}^{*}\right)
$$

for every individual action $z_{j} \in Z_{j}$.

Next, we investigate the existence of partial cooperative equilibria in arbitrary generalised partial cooperative games.

Theorem 2.3 Let $\Gamma=\langle C, N, X, Y, Z, u, v, \Lambda\rangle$ be a generalised partial cooperative game, such that

(i) All action sets $X_{i}(i \in C), Z_{j}(j \in N)$ and $Y$ are compact and convex subsets of Euclidean spaces.

(ii) The payoff function $u_{i}$ is continuous on $X_{i} \times Y$, quasi-concave on $X_{i}$, and concave on $Y$ for every cooperator $i \in C$, and the payoff function $v_{j}$ is continuous and quasi-concave on $Z_{j}$ for every non-cooperator $j \in N,{ }^{2}$

(iii) The aggregator $\Lambda$ is continuous, Paretian, and quasi-concave on $\mathbb{R}^{k}$.

Then $\Gamma$ admits at least one partial cooperative equilibrium.

For a proof of Theorem 2.3, we refer to the appendix of this paper.

${ }^{2}$ Let $A$ be convex. A function $f: A \rightarrow \mathbb{R}$ is concave if for all $\lambda \in(0,1)$ and $a^{\prime}, a^{\prime \prime} \in A: f(\lambda$. $\left.a^{\prime}+(1-\lambda) \cdot a^{\prime \prime}\right) \geqslant \lambda \cdot f\left(a^{\prime}\right)+(1-\lambda) \cdot f\left(a^{\prime \prime}\right)$, and $f$ is quasi-concave if for all $\lambda \in(0,1)$ and $a^{\prime}, a^{\prime \prime} \in A: f\left(\lambda \cdot a^{\prime}+(1-\lambda) \cdot a^{\prime \prime}\right) \geqslant \min \left\{f\left(a^{\prime}\right), f\left(a^{\prime \prime}\right)\right\}$. 


\subsection{Leadership equilibrium}

Next, we implement a sequential structure on the decision-making process in generalised partial cooperative games. We consider an internal two-tier hierarchical structure in which the coalition of cooperators $C$ has a leadership position in the decision-making process. Thus, in the first instance, $C$ writes a binding agreement resulting in the selection of some collective action $y \in Y$, and, subsequently, all cooperators $i \in C$ and all non-cooperating players $j \in N$ respond to that selection by selecting private actions $x_{i} \in X_{i}$, respectively $z_{j} \in Z$.

The leadership equilibrium concept reflects a standard backward induction logic in which the coalition of cooperators $C$ acts as a Stackelberg leader. The coalition of cooperators $C$ is assumed to act from an optimistic point of view in this procedure and considers only outcomes from the second-stage interaction between all agents that correspond to the best possible outcome for them collectively.

To define the leadership equilibrium concept properly, we have to introduce some hypotheses on the fundamentals.

Axiom 2.4 Consider a generalised partial cooperative game $\Gamma=\langle C, N, X, Y, Z, u$, $v, \Lambda\rangle$. We assume that the following properties hold:

(i) For every $i \in C$, it holds that $X_{i}$ is a compact and convex subset of some Euclidean space.

(ii) For every $j \in N$, it holds that $Z_{j}$ is a compact and convex subset of some Euclidean space.

(iii) The set of collective actions $Y$ is a compact subset of some Euclidean space.

(iv) For every cooperator $i \in C$, the payoff function $u_{i}: A \rightarrow \mathbb{R}$ is a continuous function and the section $u_{i}\left(\cdot, x_{-i}, y, z\right): X_{i} \rightarrow \mathbb{R}$ is quasi-concave on $X_{i}$ for all $\left(x_{-i}, y, z\right) \in X_{-i} \times Y \times Z$

(v) For every non-cooperator $j \in N$, the payoff function $v_{j}: a \rightarrow \mathbb{R}$ is a continuous function and the section $v_{j}\left(x, y, \cdot, z_{-j}\right)$ is quasi-concave on $Z_{j}$ for all $\left(x, y, z_{-j}\right) \in X \times Y \times Z_{-j}$.

(vi) The aggregator $\Lambda$ is continuous on $\mathbb{R}^{k}$.

The assumptions introduced in Axiom 2.4 are weaker than the ones imposed in Theorem 2.3.

Under Axiom 2.4, we can now formulate some auxiliary notations that are required for the definition of the leadership equilibrium concept. For any given collective action $y \in Y$, we denote by $\Gamma_{y}=\left\langle C \cup N, X \times Z, w^{y}\right\rangle$ a standard normal-form game given by player set $C \cup N$ and payoff functions $w_{i}^{y}$ for all players in $C \cup N$ that are described as follows:

- First, for every cooperator $i \in C$, we consider the conditional payoff function $w_{i}^{y}: X \times Z \rightarrow \mathbb{R}$ given by

$$
w_{i}^{y}(x, z)=u_{i}(x, y, z) .
$$


- Second, for every non-cooperating player $j \in N$, the given action set $Z_{j}$ as well as a conditional payoff function $w_{j}^{y}: X \times Z \rightarrow \mathbb{R}$ is defined as follows:

$$
w_{j}^{y}(x, z)=v_{j}(x, y, z) .
$$

The non-cooperative normal-form game $\Gamma_{y}=\left\langle C \cup N, X \times Z, w^{y}\right\rangle$ is denoted as the conditional partial cooperative game for the collective action $y \in Y$. The set of Nash equilibria of the conditional game $\Gamma_{y}$ is now denoted by $E_{y} \subset X \times Z$. Under the given hypotheses, this definition is non-trivial:

Lemma 2.5 Consider a generalised partial cooperative game $\Gamma=\langle C, N, X, Y, Z, u$, $v, \Lambda\rangle$ satisfying Axiom 2.4. Then, for every collective action $y \in Y$, the set of Nash equilibria $E_{y}$ of the conditional partial cooperative game $\Gamma_{y}=\left\langle C \cup N, X \times Z, w^{y}\right\rangle$ forms a non-empty, compact subset of the (Euclidean) strategy set $X \times Z$.

Lemma 2.5 is an immediate corollary from the more general assertion stated as Lemma A. 2 in the Appendix of this paper.

We postulate that the cooperators have an optimistic outlook on their abilities to gain from their leadership position. ${ }^{3}$ Using the definition of $U$ given in (2) and the assumptions made in Axiom 2.4, we let for every $y \in Y$,

$$
\begin{aligned}
U^{s}(y) & =\max _{(x, z) \in E_{y}} U(x, y, z) \\
\Phi^{s} & =\arg \max U^{s} \equiv\left\{y^{\prime} \in Y \mid U^{s}\left(y^{\prime}\right)=\max _{y \in Y} U^{s}(y)\right\} .
\end{aligned}
$$

The function $U^{s}$ assigns the maximum payoff level to the coalition of cooperators $C$ that can be achieved when the cooperators as well as the non-cooperators do a best response in their respective private and individual actions to all players' actions. ${ }^{4}$

The set $\Phi^{s}$ of $U^{s}$-maximisers describes the best responses of the coalition of cooperators $C$ to the other players' actions. Indeed, $\Phi^{s}$ is the collection of collective actions that maximise the maximum payoff envelope function $U^{s}$ over $Y$, i.e., these collective actions are coalition $C^{\prime}$ 's best responses given the collective payoff function $U^{s}$.

We can now define the leadership equilibrium concept for $\Gamma$ as follows:

Definition 2.6 Let $\Gamma=\langle C, N, X, Y, Z, u, v, \Lambda\rangle$ be a generalised partial cooperative game that satisfies Axiom 2.4. An action tuple $\left(x^{\star}, y^{\star}, z^{\star}\right) \in A$ is a leadership equilibrium in $\Gamma$ if $y^{\star} \in \Phi^{S}$, such that $E_{y^{\star}} \neq \varnothing$ and, furthermore, that

$$
\left(x^{\star}, z^{\star}\right) \in \arg \max _{(x, z) \in E_{y^{\star}}} U\left(x, y^{\star}, z\right),
$$

where $U$ is defined as in (2).

\footnotetext{
3 We remark that this optimistic outlook can be replaced by other approaches such as a minimax logic or even a fully pessimistic outlook.

4 These concepts are, indeed, properly defined given the assumptions on $u_{i}, i \in C$, and $\Lambda$ stated in Axiom 2.4 and the compactness of $E_{y}$ from Lemma 2.5 .
} 
The existence of a leadership equilibrium in a partial cooperative game can be established under the assumed conditions.

Theorem 2.7 Let $\Gamma=\langle C, N, X, Y, Z, u, v\rangle$ be a generalised partial cooperative game that satisfies Axiom 2.4. Then, there exists at least one leadership equilibrium in $\Gamma$.

For a proof of Theorem 2.7, we again refer to the Appendix.

\subsection{Separability}

A special class of generalised partial cooperative games is characterised by the separation of decisions concerning: on one hand, the collective actions $(y)$ and, on the other hand, the private $(x)$ as well as individual $(z)$ actions. Formally, a generalised partial cooperative game $\Gamma=\langle C, N, X, Y, Z, u, v, \Lambda\rangle$ is called separable if $\Lambda=\Lambda^{u}$ is the utilitarian aggregator and for every $i \in C$ and $(x, y, z) \in X \times Y \times Z$ :

$$
u_{i}(x, y, z)=\hat{u}_{i}(x, z)+\tilde{u}_{i}(y)
$$

where $\hat{u}_{i}: X \times Z \rightarrow \mathbb{R}$ and $\tilde{u}_{i}: Y \rightarrow \mathbb{R}$ are partial payoff functions.

Separability guarantees that the two notions of equilibrium defined here for the class of generalised partial cooperative games coincide. Indeed, in a separable game, the collective decision of coalition $C$ concerning the collective actions $y$ is completely described by the maximisation of $\tilde{U}=\sum_{i \in C} \tilde{u}_{i}$ and, therefore, is strictly independent of the selection of individual actions of the cooperators $i \in C$. This implies immediately the following assertion.

Proposition 2.8 Let $\Gamma$ be a separable generalised partial cooperative game satisfying Axiom 2.4. Then, the notion of leadership equilibrium is equivalent to that of the partial cooperative equilibrium concept in $\Gamma$ in the sense that every partial cooperative equilibrium is a leadership equilibrium.

In a separable partial cooperative game with multi-dimensional collective actions in the sense that $Y=\prod_{i \in C} Y_{i}$ for appropriately chosen $Y_{i}$, one can also introduce the notion of a standard Nash equilibrium. This notion is rather useful in the analysis of the effects of collective decision-making on certain specified actions.

Definition 2.9 An action tuple $\left(x^{\prime}, y^{\prime}, z^{\prime}\right)$ is a Nash equilibrium in a separable partial cooperative game $\Gamma$ with $Y=\prod_{i \in C} Y_{i}$ if, for every cooperator $i \in C$, the pair $\left(x_{i}^{\prime}, y_{i}^{\prime}\right)$ maximises $u_{i}\left(\cdot, x_{-i}^{\prime}, \cdot, y_{-i}^{\prime}, z^{\prime}\right)$ over $X_{i} \times Y_{i}$, and for every non-cooperator $j \in N$, the individual action $z_{j}^{\prime}$ maximises $v_{j}\left(x^{\prime}, y^{\prime}, \cdot, z_{-j}^{\prime}\right)$ over $Z_{j}$.

In general, the class of Nash equilibria is different from the class of Partial Cooperative and Leadership equilibria in these separable games. This is shown through the following example, which considers a separable generalised partial cooperative game with two collaborators.

Example 2.10 Consider a separable partial cooperative game $\Gamma$ with $C=\{1,2\}$ and $N$ arbitrary. We only specify the payoffs resulting from the collective actions and let

$$
\tilde{u}_{1}\left(y_{1}, y_{2}\right)=-\left(y_{1}-1\right)^{2}+2 y_{2}-3
$$




$$
\tilde{u}_{2}\left(y_{1}, y_{2}\right)=2 y_{1}-3-\left(y_{2}-1\right)^{2} \text {. }
$$

Then, in the Nash equilibrium for this game, we have that $y_{1}^{\prime}=y_{2}^{\prime}=1$ regardless of the payoff functions and the strategies of all other players. On the other hand, in the partial cooperative equilibrium, $\left(y_{1}, y_{2}\right)$ maximises

$$
\tilde{U}\left(y_{1}, y_{2}\right)=\tilde{u}_{1}\left(y_{1}, y_{2}\right)+\tilde{u}_{2}\left(y_{1}, y_{2}\right)=-\left(y_{1}-2\right)^{2}-\left(y_{2}-2\right)^{2} \text {. }
$$

This implies that, in the PCE, we arrive at $y_{1}^{*}=y_{2}^{*}=2$, again regardless of the actions of all other players in the game.

We refine the notion of separability further to arrive at some further conclusions concerning the equivalence of Nash equilibria and the other equilibrium concepts. Specifically, a generalised partial cooperative game $\Gamma=\langle C, N, X, Y, Z, u, v, \Lambda\rangle$ is called homogeneous if $\Gamma$ is separable, such that $Y=\prod_{i \in C} Y_{i}$ is multi-dimensional and for every cooperator $i \in C$ and every action tuple $(x, y, z) \in X \times Y \times Z$ :

$$
u_{i}(x, y, z)=\hat{u}_{i}(x, z)+\tilde{u}(y),
$$

where $\hat{u}_{i}: X \times Z \rightarrow \mathbb{R}$ and $\tilde{u}: Y \rightarrow \mathbb{R}$.

We can now state the following assertion without proof. A proof is based on the insight that the determination of the collective action $y \in Y=\prod_{i \in C} Y_{i}$ is not only completely separated from the determination of the other actions $(x, z) \in X \times Z$, but that it is essentially based on the maximisation of the common payoff function $\tilde{u}$. Thus, the optimal collective action coincides with the individually optimal choice of $y_{i} \in Y_{i}$ for every cooperator $i \in C$.

Proposition 2.11 Let $\Gamma$ be a homogeneous generalised partial cooperative game. Then, the notion of partial cooperative equilibrium is equivalent to that of Nash equilibrium in $\Gamma$ in the sense that every partial cooperative equilibrium is a Nash equilibrium.

\section{Two applications}

In this section, we investigate two applications of generalised partial cooperative games that clearly delineate the various equilibrium concepts. Both of these applications consider a generalised partial cooperative game formulation that allows the development of a standard Nash equilibrium. Hence, the concept of a standard Nash equilibrium is well defined and can be computed. Thus, a complete comparison of these Nash equilibria with the two main partial cooperative equilibrium concepts-partial cooperative equilibrium and leadership equilibrium—can be developed.

\subsection{Cartels in multi-market oligopolies}

We first consider a cartel in a multi-market oligopoly. Firms are fully competitive in one market, while a subset of firms forms a cartel in the second market only. We show 
that the cartel benefits in comparison with the standard Nash equilibrium outcome if it has a clear leadership position: An improvement only occurs in the leadership equilibrium, not in the partial cooperative equilibrium.

We limit our analysis to the case of three firms indexed by $i=1,2,3$ that compete in two related markets $A$ and $B .{ }^{5}$ We assume that these firms engage in quantitysetting, Cournotian competition. We denote by $q_{i}$ and $Q_{i}$ the quantities sold by firm $i$ on markets $A$ and $B$, respectively. Furthermore, $p$ and $P$ represent the market prices emerging in market $A$ and $B$, respectively.

Focusing on the most interesting case, we suppose that competitors' products are strategic substitutes and there are diseconomies of scope across the two markets. More specifically, demand in markets $A$ and $B$ for firm $i$ is, respectively, represented by

$$
p=\alpha-\sum_{i=1}^{m} q_{i} \quad \text { and } \quad P=\beta-\sum_{i=1}^{m} Q_{i}
$$

where $\alpha, \beta>0$ are demand parameters describing the total market size for each of the two goods $A$ and $B$.

We assume a well-established, widely adopted production technology imposing a simple, identical quadratic cost function on the three firms, given by

$$
C(q, Q)=\frac{1}{2}(q+Q)^{2}
$$

Therefore, the profit of firm $i$ is determined as

$$
\pi_{i}=p \cdot q_{i}+P \cdot Q_{i}-C\left(q_{i}, Q_{i}\right)=p \cdot q_{i}+P \cdot Q_{i}-\frac{1}{2}(q+Q)^{2} .
$$

It can be shown that, in this linear quadratic formulation, the resulting second-order conditions are always satisfied if $\alpha$ and $\beta$ are not too different. Hence, one obtains a unique interior maximum through the first-order conditions.

\section{Formulation as a generalised partial cooperative game}

We set up a generalised partial cooperative game that describes cartel formation in this multi-market Cournot oligopoly. We consider the formation of the cartel $C=\{1,2\}$ only in market $B$, while full competition is retained in market $A$. Here, firm 3 remains an independent producer. ${ }^{6}$

In this multi-market framework, $y=\left(Q_{1}, Q_{2}\right)$ forms the collective action of the cartel $C=\{1,2\}$, while quantities $\left(q_{1}, q_{2}\right)$ set in the $A$-market constitute private actions for the two cartel members $i=1,2$. Firm 3 acts completely independently in both markets and set all output levels $\left(q_{3}, Q_{3}\right)$ as individual actions.

\footnotetext{
5 Throughout, we use the notation of Billand et al. (2014).

6 We remark that our analysis can be extended to an arbitrary number of competitors and cartel members. This generalization, however, leads to rather cumbersome expressions that add rather little to the conclusions from our analysis. The case for any number of competitors is available upon request from the authors.
} 
The generalised partial cooperative game is completed with the selection of the utilitarian aggregator $\Lambda^{u}\left(\pi_{1}, \pi_{2}\right)=\pi_{1}+\pi_{2}$ to direct the collective decisions of the cartel $C=\{1,2\}{ }^{7}$

\section{Equilibrium analysis}

In this setting, we discuss the Nash equilibrium, the partial cooperative equilibrium, and the leadership equilibrium in this model of cartel formation in a multi-market oligopoly.

We take Nash equilibrium as a benchmark with respect to which we compare the results of the two partial cooperative analysis. If all three firms act competitively and $\left(q_{i}, Q_{i}\right), i=1,2,3$, are set independently, then a straightforward analysis shows that a unique symmetric Nash equilibrium exists if $\frac{\alpha}{5}<\beta<5 \alpha$. This equilibrium is given by

$$
\begin{gathered}
q_{1}^{N E}=q_{2}^{N E}=q_{3}^{N E}=\frac{5 \alpha-\beta}{24} \equiv q^{N E} \\
Q_{1}^{N E}=Q_{2}^{N E}=Q_{3}^{N E}=\frac{5 \beta-\alpha}{24} \equiv Q^{N E} .
\end{gathered}
$$

In the symmetric Nash equilibria, all firms earn equal profits:

$$
\pi_{1}^{N E}=\pi_{2}^{N E}=\pi_{3}^{N E}=\frac{17 \alpha^{2}+17 \beta^{2}-2 \alpha \beta}{288} \equiv \pi^{N E} .
$$

\section{Partial cooperative and leadership equilibria}

Next, we investigate the partial cooperative and leadership equilibria in this generalised partial cooperative game.

Proposition 3.1 Given a multi-sided oligopoly with firm profit functions given by (14), then

- A Partial cooperative equilibrium exists and is unique if $\frac{\alpha}{5}<\beta<5 \alpha$ and is given by

$$
\begin{gathered}
q_{1}^{P E}=q_{2}^{P E}=\frac{20 \alpha-2 \beta}{98} ; \quad q_{3}^{P E}=\frac{21 \alpha-7 \beta}{98} ; \\
Q_{1}^{P E}=Q_{2}^{P E}=\frac{15 \beta-3 \alpha}{98} ; \quad Q_{3}^{P E}=\frac{25 \beta-5 \alpha}{98} .
\end{gathered}
$$

- A unique leadership equilibrium exists and is given by

$$
q_{1}^{L E}=q_{2}^{L E}=\frac{205 \alpha-37 \beta}{968} ; \quad q_{3}^{L E}=\frac{197 \alpha-45 \beta}{968} ;
$$

\footnotetext{
7 We mention here that replacing the utilitarians aggregator by the Rawlsian aggregator $\Lambda^{r}=\min \left\{\pi_{1}, \pi_{2}\right\}$ would not affect the analysis of this particular application. In particular, this is due to the symmetry of the described situation.
} 


$$
Q_{1}^{L E}=Q_{2}^{L E}=\frac{193 \beta-49 \alpha}{968} ; \quad Q_{3}^{L E}=\frac{19 \beta-3 \alpha}{88} .
$$

Deriving the above results involves straightforward albeit tedious calculations and thus are not presented here. ${ }^{8}$ We compute that

$$
\begin{aligned}
\pi_{1}^{P E}=\pi_{2}^{P E} & =\frac{1125 \alpha^{2}+1077 \beta^{2}-78 \alpha \beta}{19208} \text { and } \\
\pi_{3}^{P E} & =\frac{1188 \alpha^{2}+1672 \beta^{2}-512 \alpha \beta}{19208}
\end{aligned}
$$

for the partial equilibrium case, and

$$
\begin{aligned}
\pi_{1}^{L E}=\pi_{2}^{L E} & =\frac{229 \alpha^{2}+229 \beta^{2}-26 \alpha \beta}{3872} \text { and } \\
\pi_{3}^{L E} & =\frac{26673 \alpha^{2}+29557 \beta^{2}-2314 \alpha \beta}{468512} .
\end{aligned}
$$

for the leadership equilibrium.

For $i=1,2$, we conclude that

$$
\pi_{i}^{L E} \geqslant \max \left\{\pi_{i}^{P E}, \pi_{i}^{N E}\right\}
$$

We can now also investigate whether the merger paradox, seminally stated for Cournot competition in Salant et al. (1983) emerges in any of these environments. The merger paradox would occur if cartel formation in this multi-market setting results into losses to its members. Clearly, (15) implies that there are no grounds for merger paradox to emerge if the cartel obtains a leadership position in the multi-market oligopoly, and, that instead there may be market conditions that make it a feature of the partial cooperative equilibrium when $\pi_{i}^{P E}<\pi_{i}^{N E}$ for the cartel members $i=1,2$. Here, that is the case for $\beta>\frac{317}{409} \alpha \approx 0.775 \alpha$. Thus, the merger paradox occurs if market $B$ is sufficiently larger than the competitive market $A$. On the other hand, forming a cartel on a relatively smaller market may result in a strict Pareto improvement. We compute that this is particularly the case for $\frac{1951}{3875} \alpha<\beta<\frac{317}{409} \alpha$.

We can illustrate this point more clearly by presenting the equilibrium outcome for specific values of the market parameters. For example, if $\alpha=5$ and $\beta=3$, we arrive at $\pi_{1}^{P E}=\pi_{2}^{P E}=1.9080>\pi^{N E}=1.9028$ as well as $\pi_{3}^{P E}=1.9298>$ $\pi^{N E}=1.9028$. Here, a leadership role improves the profit position for the cartel, although the independent producer firm 3 still outperforms its competitors. To be precise, we compute that $\pi_{1}^{L E}=\pi_{2}^{L E}=1.9101>\pi_{1}^{P E}=\pi_{2}^{P E}>\pi^{N E}$, while $\pi_{3}^{P E}>\pi_{3}^{L E}=1.9170>\pi^{N E}$.

8 Detailed derivations are available from the authors upon request. 


\subsection{International pollution abatement}

Our second application concerns an international environmental protection situation in which certain countries write an international pollution abatement treaty, while other countries do not participate in such a collective control of their emissions. Our analysis builds on the seminal contribution by Chander and Tulkens (1997) who, for the purpose of their analysis, developed the notion of the $\gamma$-core for partition function form games. We remark here that their notion of partial agreement equilibrium corresponds to our notion of partial cooperative equilibrium.

We amend their analysis by supplementing the cooperators' strategy space with an additional dimension along which binding agreements and cooperation are not possible. Therefore, we include an input factor-denoted as labour-that treaty countries choose independently, similarly to non-cooperators.

There are two types of countries, namely those that cooperate on writing an international emissions control treaty-denoted by $C=\{1, \ldots, k\}$ with $k \geqslant 2$-and those that act independently-denoted by $N=\{1, \ldots, n\}$ with $n \geqslant 1$. All countries produce two non-tradable goods, 1 and 2, only. Treaty country $i \in C$ produces and consumes quantities $q_{1 i}$ and $q_{2 i}$, while non-treaty country $j \in N$ produces and consumes $q_{1 j}$ and $q_{2 j}$, respectively.

Good 1 is "clean" in the sense that its production leaves no emissions footprint. Good 2 is "dirty" in the sense that its production requires an input that leaves an emissions footprint. For all countries, the production technology is the same. For generic country $h \in C \cup N$, the production functions for the two goods are given by $q_{1 h}=\sqrt{L_{1 h}}$ and $q_{2 h}=\sqrt{d_{h} L_{2 h}}$, where $L_{1 h} \geqslant 0$ and $L_{2 h} \geqslant 0$ are, respectively, the amounts of labour expended in the production of goods 1 and 2, and $d_{h} \geqslant 0$ is the "dirty input" in the production of good 2.

We further assume that every country $h \in C \cup N$ is endowed with the same total labour input equal to $\bar{L}$. Thus, $L_{1 h}+L_{2 h} \leq \bar{L}$. Throughout, we assume that $\bar{L}>1$.

The utility function of a representative consumer of country $h \in C \cup N$ is now given by

$$
u_{h}\left(q_{1 h}, q_{2 h}, \Delta\right)=q_{1 h}+q_{2 h}-\frac{1}{2} \Delta
$$

with

$$
\begin{aligned}
\Delta & =\sum_{i=1}^{k} d_{i}+\sum_{j=1}^{n} d_{j} \\
q_{1 h} & =\sqrt{L_{1 h}} \\
q_{2 h} & =\sqrt{d_{h} L_{2 h}} \\
L_{1 h}+L_{2 h} & \leqslant \bar{L} .
\end{aligned}
$$


Here, $\Delta$ represents the emissions associated with the production of good 2 across the global community. ${ }^{9}$ Note that this payoff function is separable in the production variables $\left(q_{1 h}, q_{2 h}\right)$ on one hand and the emission decisions $\left\{d_{h}\right\}_{h \in C \cup N}$ on the other. This implies that the generalised partial cooperative game considered here is separable and Proposition 2.8 applies.

In terms of the notation employed, $y=\left(d_{1}, \ldots, d_{k}\right)$ is the multi-dimensional collective action of all treaty countries in $C$, representing the treaty written between the countries in $C$ to control emissions. Throughout, we postulate that the coalition of treaty countries $C$ is guided by utilitarian principles and uses the utilitarian aggregator $\Lambda^{u}=\sum_{i \in C} u_{i}$ to guide its collective decisions.

\section{Equilibrium analysis}

We can approach this model as a normal-form strategic game as well as a generalised partial cooperative game. We first determine its Nash equilibrium that we use as a benchmark. This represents a situation in which all countries in $C$ act independently and do not write a treaty to control their emissions. This results into a standard tragedy of the commons problem which is given by the maximisation of the objective function (16) subject to the constraints listed in (17)-(20).

Indeed, in the Nash equilibrium for each country $h \in C \cup N$, we derive that $L_{1 h}^{N E}=1$ and $L_{2 h}^{N E}=d_{h}^{N E}=\bar{L}-1$, resulting into $u_{h}^{N E}=\bar{L}-\frac{1}{2}(k+n)(\bar{L}-1){ }^{10}$

\section{Partial cooperative and leadership equilibria}

First, note that the formulated generalised partial cooperative game is separable and that Proposition 2.8 now implies that the partial cooperative equilibria are equal to the leadership equilibria in this model. We, therefore, limit our discussion to the partial cooperative equilibria only.

In the case of cooperation, the coalition of treaty countries $C$ signs a binding agreement $y=\left(d_{1}, \ldots, d_{k}\right)$ and each country $h \in C \cup N$ chooses independently the amount of labour that it allocates to the production of each of the two goods $\left(L_{1 h}, L_{2 h}\right)$. Moreover, each non-treaty country $j \in N$ makes a decision on the dirty input $d_{j}$.

The coalition of treaty countries $C$ determines $y=\left(d_{1}, \ldots, d_{k}\right)$ by solving to the following maximisation problem:

$$
\max _{d_{1}, \ldots, d_{k}} \sum_{i \in C}\left(\sqrt{\bar{L}-L_{2 i}}+\sqrt{d_{i} L_{2 i}}-\frac{1}{2}\left(\sum_{i=1}^{k} d_{i}+\sum_{j=1}^{n} d_{j}\right)\right) .
$$

\footnotetext{
9 To bring to the fore the mechanism of partial cooperation, we take countries to be completely homogeneous and goods to be non-tradable. As a consequence, all countries remain in a state of autarky.

10 Obviously, $u^{N E}<0$ if the total number of countries $k+n \geqslant 3(\geqslant 4)$ and the total in-country labour input $\bar{L}>3(>2)$. In that case, the negative impact of pollution overtakes the positive utility from the consumption of the two goods.
} 
This yields that for every $i \in C: d_{i}=\frac{L_{2 i}}{k^{2}}$. This now results in the following conclusions: ${ }^{11}$

Proposition 3.2 There are two cases for which a complete description of the partial cooperative equilibrium of the pollution abatement model can be determined:

(a) $1<\bar{L} \leqslant k^{2}$ : For each treaty country, $i \in C: L_{1 i}^{P E}=\bar{L}$ and $L_{2 i}^{P E}=d_{i}^{P E}=0$. For each independent country $i \in N: L_{1 j}^{P E}=1$ and $L_{2 j}^{P E}=d_{j}^{P E}=\bar{L}-1$. The total pollution in this equilibrium is determined as $\Delta^{P E}=n(\bar{L}-1)$ and the resulting utility levels as

$$
\begin{aligned}
& u_{i}^{P E}=\sqrt{\bar{L}}-\frac{n}{2}(\bar{L}-1) \\
& u_{j}^{P E}=\left(1-\frac{n}{2}\right) \bar{L}+\frac{n}{2} .
\end{aligned}
$$

For all $1<\bar{L} \leqslant k^{2}$, the partial cooperative equilibrium is a strict Pareto improvement over the Nash equilibrium.

(b) $\bar{L}>k^{2}:$ For each treaty country, $i \in C: L_{1 i}^{P E}=k^{2}, L_{2 i}^{P E}=\bar{L}-k^{2}$ and $d_{i}^{P E}=$ $\frac{\bar{L}-k^{2}}{k^{2}}$. For each independent country $i \in N: L_{1 j}^{P E}=1$ and $L_{2 j}^{P E}=d_{j}^{P E}=\bar{L}-1$. The total pollution in this equilibrium is determined as

$$
\Delta^{P E}=\left(n+\frac{1}{k}\right) \bar{L}-(k+n),
$$

and the resulting utility levels as

$$
\begin{aligned}
& u_{i}^{P E}=\frac{\bar{L}-k^{2}}{k}+\frac{1}{2}(3 k+n)-\frac{1}{2}\left(n+\frac{1}{k}\right) \bar{L} \\
& u_{j}^{P E}=\bar{L}-\frac{1}{2}\left(n+\frac{1}{k}\right) \bar{L}+\frac{1}{2}(k+n) .
\end{aligned}
$$

For any $\bar{L}>k^{2}$, the partial cooperative equilibrium is a strict Pareto improvement over the Nash equilibrium.

The conclusion from Proposition 3.2 is that treaty countries as well as independent countries are better-off under pollution abatement treaties than under global noncollaboration as described in the Nash equilibrium. Although, in Nash equilibrium, all countries achieve the same utility levels, under partial cooperation, the independent countries have a higher utility than the cooperators, since they act as free riders, enjoying the environmental pollution abatement imposed through the treaty. As expected, total pollution always increases with the number of independent countries. An increase in the number of treaty countries, on the other hand, decreases total pollution.

\footnotetext{
11 The computations and derivations are rather tedious and, therefore, relegated to the appendix of this paper.
} 


\section{Concluding remarks}

In this paper, we introduce two equilibrium notions that are applicable to situations of partial cooperation. We establish their existence properties in a general and widely applicable setting.

First, as our concept of partial cooperative equilibrium builds on the standard notion of Nash equilibrium, it is neutral to the assumption of observability of players' strategies, including the existence and composition of the coalition of cooperators.

Second, even though our results are derived in the context of a single group of cooperators, our results on the partial cooperative equilibrium concept are generalisable to a setting with an arbitrary number of exclusive coalitions of cooperators. For a partial cooperative equilibrium to exist in such more general settings, the conditions on the aggregator function identified in Theorem 2.3 must apply to all coalitions of cooperators.

In the context of leadership in partial cooperation, we present our results under the assumption that the cooperators have a leadership role. A similar existence result can be derived if, instead, one of the non-cooperators acts as the Stackelberg leader. To see that, it is sufficient to recognise that once the first mover takes action, the players that are moving next are de facto in a situation of partial cooperation, where the existence of a fixed point is established in Theorem 2.3.

Finally, we point out the role of complete information in our set up. Here, as in the standard Nash Equilibrium analysis, we take complete information to implicitly imply common knowledge of payoffs. In the context of leadership equilibrium, we make an implicit requirement that the collective action is observable by all players, too.

\section{Possible extensions of our analysis}

Despite its robustness, we acknowledge that our analysis is restrictive in the sense that cooperation between the coalition members is ex-ante postulated and binding. In this respect, our work is complementary to the large literature on the endogenous formation of agreements based on the work of Ichiishi (1981), Bloch (1997), and Yi (1997). ${ }^{12}$ Whereas the focus of this literature is on the existence and stability of a stable coalition structure given a payoff allocation, our contribution is on the equilibrium derivation of payoffs in a strategic environment taking the coalition structure as given. While, in these contexts, the existence of equilibria is not an issue, our contribution guides future research on the entire class of games where equilibrium existence is guaranteed by deriving very general conditions on the payoff functions. Clearly, further effort is needed in the direction of studying the general properties of the simultaneous game of coalition formation and partial cooperation.

In the context of the two specific applications developed here, an indication of coalition stability can be derived from the comparison between the partial equilibrium payoffs and those in the Nash Equilibrium. If one takes Nash equilibrium to be resulting when cooperation breaks down, then conditions under which partial (lead-

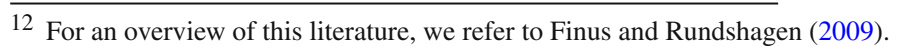


ership) equilibrium payoffs for the cooperators are higher are suggestive of the range of conditions necessary to sustain cooperative agreements.

The two applications discussed also underline both the significance and the relevance of the equilibrium notions based on partial cooperation. In particular, in our first application, we embed a standard framework of multi-market oligopolistic competition into our analysis of partial cooperation. By doing so, we generalise the analysis in existing studies - e.g., Bernheim and Whinston (1990) and more recently Choi and Gerlach (2013) - that analyse the profitability of cartels in multi-market context. In this literature, cartels are of limited size and occur in the absence of outside competitive pressures. Our analysis suggests that, when firms meet on two markets, cartels are more likely to be stable on the relatively smaller market, or where the cartel can take a leadership position relative to non-cartel members. To the best of our knowledge, we are the first to point out the advantages that cartels attain in multi-market oligopolies in the context of sequential decision-making.

Our second application concerning environmental agreements draws out the role of separability in the analysis. As discussed in the introduction, partial cooperation in this context has been studied since the 1990s. The main conclusion of our analysis-that payoffs for the signatories are equal under partial cooperative equilibrium and under leadership equilibrium - points to the fact that arguments that a success of partial cooperation is dependent on taking a first-mover advantage are not always justifiable.

Open Access This article is distributed under the terms of the Creative Commons Attribution 4.0 International License (http://creativecommons.org/licenses/by/4.0/), which permits unrestricted use, distribution, and reproduction in any medium, provided you give appropriate credit to the original author(s) and the source, provide a link to the Creative Commons license, and indicate if changes were made.

\section{A Appendix: Proofs of theorems and propositions}

This appendix collects the proofs of the main existence theorems presented in Sect. 2 of this paper.

\section{A.1 Proof of Theorem 2.3}

For every cooperator $i \in C$, we define her best response correspondence as a mapping $\gamma_{i}: X_{-i} \times Y \times Z \rightarrow 2^{X_{i}}$ given by

$$
\gamma_{i}\left(x_{-i}, y, z\right)=\underset{x_{i} \in X_{i}}{\arg \max } u_{i}\left(x_{i}, x_{-i}, y, z\right)
$$

for any $\left(x_{-i}, y, z\right) \in X_{-i} \times Y \times Z$.

Similarly, for the coalition of cooperators $C$ itself, we define the collective best response correspondence $\delta: X \times Z \rightarrow 2^{Y}$ by

$$
\delta(x, z)=\underset{y \in Y}{\arg \max } U(x, y, z)
$$


for any $(x, z) \in X \times Z$, where $U$ is the aggregated payoff function for $C$ defined in (2).

Furthermore, for every non-cooperator $j \in N$, we define her best response correspondence $\varepsilon_{j}: X \times Y \times Z_{-j} \rightarrow 2^{Z_{j}}$ by

$$
\varepsilon_{j}\left(x, y, z_{-j}\right)=\underset{z_{j} \in Z_{j}}{\arg \max } v_{j}\left(x, y, z_{j}, z_{-j}\right)
$$

for any $\left(x, y, z_{-j}\right) \in X \times Y \times Z_{-j}$.

This allows us to introduce the joint best response correspondence $\mathbf{B}: A \rightarrow 2^{A}$, such that for any $\hat{a}=(\hat{x}, \hat{y}, \hat{z}) \in A$ :

$$
\mathbf{B}(\hat{a})=\left\{\begin{array}{l|l}
(x, y, z) \in A & \begin{array}{l}
x_{i} \in \gamma_{i}(\hat{x}-i, \hat{y}, \hat{z}) \\
y \in \delta(\hat{x}, \hat{z}), \quad \text { and } \\
z_{j} \in \varepsilon_{j}\left(\hat{x}, \hat{y}, \hat{z}_{-j}\right)
\end{array} \text { for all } i \in C, \\
\text { for } j \in N
\end{array}\right\} .
$$

It is clear that a fixed point of this best response correspondence-defined as some $a^{\star} \in A$, such that $a^{\star} \in \mathbf{B}\left(a^{\star}\right)$-corresponds to a partial cooperative equilibrium of the generalised partial cooperative game $\Gamma$.

We proceed by showing that $\mathbf{B}$, indeed, possesses such a fixed point.

First, we show that the best response correspondences $\gamma_{i}(i \in C), \delta$, and $\varepsilon_{j}(j \in N)$ are all non-empty valued.

Given that $X_{i}$ is compact and $u_{i}$ continuous on $X_{i}$, for every $i \in C$, applying the Weierstrass Theorem implies that $u_{i}$ indeed admits a maximum and, thus, $\gamma_{i}\left(x_{-i}, y, z\right) \neq \varnothing$ for all $a=(x, y, z) \in A$, where $i \in C$.

Next, since the aggregator $\Lambda$ is continuous and all $u_{i}, i \in C$, are continuous on $Y$, it follows that $U$ is continuous on $Y$ as well. From compactness of $Y$, it follows that $\delta$ is, therefore, non-empty valued.

Finally, the compactness of $Z_{j}$ and continuity of $v_{j}$ on $Z_{j}$, for all $j \in N$, implies that $\varepsilon_{j}$ is non-empty valued as well for all $j \in N$.

Therefore, combining these facts, it follows that $\mathbf{B}$ is a non-empty valued correspondence.

Next, we show that $\mathbf{B}$ is convex-valued.

First, we claim that each of the correspondences $\gamma_{i}(i \in C), \delta$, and $\varepsilon_{j}(j \in N)$ is convex-valued. To see this, consider $\gamma_{i}$ for some $i \in C$. For any $\left(x_{-i}, y, z\right) \in X_{-i} \times$ $Y \times Z, \gamma_{i}\left(x_{-i}, y, z\right)$ is the set of maxima of the quasi-concave function $u_{i}\left(\cdot, x_{-i}, y, z\right)$ mapped onto the convex set $X_{i}$. Hence, $\gamma_{i}\left(x_{-i}, y, z\right)$ is, indeed, a convex set.

Similar arguments can be used to show that, for any $\left(x, y, z_{-j}\right) \in X \times Y \times Z_{-j}$, the set $\varepsilon_{j}\left(x, y, z_{-j}\right)$ is convex.

Finally, consider $\delta$. Since $\Lambda$ is Paretian and quasi-concave, it aggregates quasiconcave utility functions in a quasi-concave function. ${ }^{13}$ Therefore, $U$ is quasi-concave on $Y$.

Furthermore, for every $(x, z) \in X \times Z, \delta(x, z)$ is the set of maxima of the quasiconcave function $U(x, \cdot, z)$ mapped on a convex set $Y$, implying that $\delta(x, z)$ is, indeed, convex.

\footnotetext{
13 We refer to Negishi (1963, Proposition1) for a concise proof of this result.
} 
This implies that $\mathbf{B}$ is, indeed, convex-valued.

Finally, we prove $\mathbf{B}$ is upper hemi-continuous.

Consider a sequence $\hat{a}_{p}=\left(\hat{x}_{p}, \hat{y}_{p}, \hat{z}_{p}\right)$ converging to some $\hat{a}=(\hat{x}, \hat{y}, \hat{z})$, as well as a sequence $\tilde{a}_{p}=\left(\tilde{x}_{p}, \tilde{y}_{p}, \tilde{z}_{p}\right)$ converging to some $\tilde{a}=(\tilde{x}, \tilde{y}, \tilde{z})$, such that $\left(\tilde{x}_{p}, \tilde{y}_{p}, \tilde{z}_{p}\right) \in$ $\mathbf{B}\left(\hat{x}_{p}, \hat{y}_{p}, \hat{z}_{p}\right)$ for all $p \in \mathbb{N}$. We now prove that $(\tilde{x}, \tilde{y}, \tilde{z}) \in \mathbf{B}(\hat{x}, \hat{y}, \hat{z})$, implying that the correspondence $\mathbf{B}$ is closed and, thus, since $A$ is compact, it follows that $\mathbf{B}$ is, indeed, upper hemi-continuous.

By definition, it follows for all $i \in C$ that for every $x_{i} \in X_{i}$ :

$$
u_{i}\left(\tilde{x}_{p, i}, \hat{x}_{p,-i}, \hat{y}_{p}, \hat{z}_{p}\right)-u_{i}\left(x_{i}, \hat{x}_{p,-i}, \hat{y}_{p}, \hat{z}_{p}\right) \geqslant 0 .
$$

For every $y \in Y$ :

$$
U\left(\hat{x}_{p}, \tilde{y}_{p}, \hat{z}_{p}\right)-U\left(\hat{x}_{p}, y, \hat{z}_{p}\right) \geqslant 0 .
$$

Finally, for every $j \in N$ and all $z_{j} \in Z_{j}$ :

$$
v_{j}\left(\hat{x}_{p}, \hat{y}_{p}, \tilde{z}_{p, j}, \hat{z}_{p,-j}\right)-v_{j}\left(\hat{x}_{p}, \hat{y}_{p}, z_{j}, \hat{z}_{p,-j}\right) \geqslant 0 .
$$

From these conclusions, it follows immediately that

$$
\begin{gathered}
u_{i}\left(\tilde{x}_{i}, \hat{x}_{-i}, \hat{y}, \hat{z}\right)-u_{i}\left(x_{i}, \hat{x}_{-i}, \hat{y}, \hat{z}\right) \geqslant 0 \text { for every } i \in C \text { and } x_{i} \in X_{i} ; \\
U(\hat{x}, \tilde{y}, \hat{z})-U(\hat{x}, y, \hat{z}) \geqslant 0 \text { for every } y \in Y ; \\
v_{j}\left(\hat{x}, \hat{y}, \tilde{z}_{j}, \hat{z}_{-j}\right)-v_{j}\left(\hat{x}, \hat{y}, z_{j}, \hat{z}_{-j}\right) \geqslant 0 \text { for every } j \in N \text { and } z_{j} \in Z_{j} .
\end{gathered}
$$

This proves that $(\tilde{x}, \tilde{y}, \tilde{z}) \in \mathbf{B}(\hat{x}, \hat{y}, \hat{z})$.

Hence, we conclude that the correspondence $\mathbf{B}: A \rightarrow 2^{A}$ is a convex-valued and upper hemi-continuous correspondence. From Kakutani's fixed point theorem (Border 1985, page 72), it can be concluded that $\mathbf{B}$ admits a fixed point and, hence, a partial cooperative equilibrium exists for the generalised partial cooperative game $\Gamma$.

\section{A.2 Proof of Theorem 2.7}

The proof of Theorem 2.7 is based on Berge's Theorem. For completeness, we state this fundamental result here.

Lemma A.1 (Berge 1997, page 115) Let $M$ and $N$ be two Euclidean spaces, and let $D: M \rightarrow 2^{N}$ be a non-empty and compact valued correspondence and $f: M \times N \rightarrow$ $\mathbb{R}$ be a upper semi-continuous function. Define the value function $V: M \rightarrow \mathbb{R}$ by

$$
V(m)=\sup \{f(n, m) \mid n \in D(m)\} .
$$

If $D$ is upper hemi-continuous at $m \in M$, then the value function $V$ is upper semicontinuous at $m$. 
Next, consider a generalised partial cooperative game $\Gamma=\langle C, N, X, Y, Z, u, v, \Lambda\rangle$. First, we define the Nash correspondence which is equal to the feasibility correspondence $E$ defined in Sect. 2 of the paper. Formally, we introduce the mapping $E: Y \rightarrow 2^{X \times Z}$ by $E(y)=E_{y}$ for every collective action $y \in Y$. We show that, under standard assumptions, this correspondence is non-empty and compact valued as well as upper hemi-continuous.

Lemma A.2 Let, for the generalised partial cooperative game $\Gamma=\langle C, N, X, Y, Z$, $u, v\rangle, X_{i}$ be a compact and convex subset of some Euclidean space for every cooperator $i \in C$ and $Z_{j}$ be a compact and convex subset of some Euclidean space for every non-cooperator $j \in N$. Assume that $X, Y$, and $Z$ are all non-empty.

Let for all $i \in C, u_{i}: A \rightarrow \mathbb{R}$ be a continuous function and $u_{i}\left(\cdot, x_{-i}, y, z\right): X_{i} \rightarrow$ $\mathbb{R}$ be quasi-concave for all $\left(x_{-i}, y, z\right) \in X_{-i} \times Y \times Z$. Furthermore, let for all $j \in N$, $v_{j}: A \rightarrow \mathbb{R}$ be a continuous function and $v_{j}\left(x, y, \cdot, z_{-j}\right)$ be quasi-concave for all $\left(x, y, z_{-j}\right) \in X \times Y \times Z_{-j}$.

Then, the correspondence $E: Y \rightarrow 2^{X \times Z}$ is non-empty and compact valued as well as upper hemi-continuous.

Proof For an arbitrary collective action $y \in Y$, consider the reduced normal-form game $\Gamma_{y}=\langle C \cup N, X \times Z, w\rangle$ as given before. Assume that $X_{i}$ is a compact and convex subset of a Euclidean space for each $i \in C$, and $Z_{j}$ is a compact and convex subset of a Euclidean space for all $j \in N$.

For any $i \in C$, the function $w_{i}^{y}$ is equal to a section of $u_{i}$ and, hence, by assumption its section $w_{i}^{y}\left(\cdot, x_{-i}, z\right)$ is quasi-concave on $X_{i}$.

For any $j \in N$, the function $w_{j}^{y}$ is equal to a section of $v_{j}$ and, consequentially, by assumption its section $w_{j}^{y}\left(x, \cdot, z_{-j}\right)$ is quasi-concave on $Z_{j}$.

Moreover, both $w_{i}^{y}(i \in C)$ and $w_{j}^{y}(j \in N)$ are continuous on $X \times Z$.

Hence, we conclude that the game $\Gamma_{y}=\langle C \cup N, X \times Z, w\rangle$ satisfies all conditions of a standard Nash equilibrium existence theorem (Fudenberg and Tirole 1991, page $34)$ and, therefore, admits a Nash equilibrium. Thus, $E_{y} \neq \varnothing$ for all $y \in Y$ implying that $E: Y \rightarrow 2^{X \times Z}$ is non-empty valued.

Next, we show that the correspondence $E$ is closed, and since $X \times Z$ is compact, $E$ is upper hemi-continuous, as well. Consider a convergent sequence $\hat{y}_{p} \rightarrow \hat{y}$ and a convergent sequence $\left(\hat{x}_{p}, \hat{z}_{p}\right) \rightarrow(\hat{x}, \hat{z})$, such that $\left(\hat{x}_{p}, \hat{z}_{p}\right) \in E\left(\hat{y}_{p}\right)$. We prove that $(\hat{x}, \hat{z}) \in E(\hat{y})$.

Indeed, by definition, for all $i \in C$, we have $w_{i}^{y}\left(\hat{x}_{p, i}, \hat{x}_{p,-i}, \hat{z}_{p}\right)-w_{i}^{y}\left(x_{i}, \hat{x}_{p,-i}, \hat{z}_{p}\right) \geqslant$ 0 for all $x_{i} \in X_{i}$. Similarly, for all $j \in N, w_{j}^{y}\left(\hat{x}_{p}, \hat{z}_{p, j}, \hat{z}_{p,-j}\right)-w_{j}^{y}\left(\hat{x}_{p}, z_{j}, \hat{z}_{p,-j}\right) \geqslant 0$ for all $z_{j} \in Z_{j}$. It follows immediately that both $w_{i}^{y}(\cdot, \cdot, \cdot)-w_{i}^{y}\left(x_{i}, \cdot, \cdot\right): X_{i} \times X_{-i} \times$ $Z \rightarrow \mathbb{R}$ and $w_{j}^{y}(\cdot, \cdot, \cdot)-w_{j}^{y}\left(\cdot, z_{j}, \cdot\right): X \times Z_{j} \times Z_{-j} \rightarrow \mathbb{R}$ are continuous functions.

Hence, for every $i \in C$, we have that $w_{i}^{y}\left(\hat{x}_{i}, \hat{x}_{-i}, \hat{z}\right)-w_{i}^{y}\left(x_{i}, \hat{x}_{-i}, \hat{z}\right) \geqslant 0$ and, for all $j \in N$, we have $w_{j}^{y}\left(\hat{x}, \hat{z}_{j}, \hat{z}_{-j}\right)-w_{j}^{y}\left(\hat{x}, z_{j}, \hat{z}_{-j}\right) \geqslant 0$. This implies $(\hat{x}, \hat{z}) \in E(\hat{y})$. Thus, $E$ is, indeed, a closed correspondence.

Finally, take any $y \in Y$. We show that $E(y)$ is compact. Since $E$ is a closed correspondence, it is, therefore, closed-valued. Hence, $E(y)$ is a closed subset of a compact set $X \times Z$ and, thus, $E(y)$ is compact. 
Next, consider the function $U^{s}: Y \rightarrow \mathbb{R}$ as defined in (8). From the continuity of $\Lambda$ and $u_{i}$ for each $i \in C$, we conclude that $U$ is continuous on $A$. Since, from Lemma A.2, the set $E_{y}$ is compact for all $y \in Y$, it then follows that $\arg \max _{(x, y) \in E_{y}} U(x, y, z)$ exists for every $y \in Y$. Hence, $U^{s}$ is a well-defined function. It remains to show that $U^{s}$ is an upper semi-continuous function.

Lemma A.3 Let the generalised partial cooperative game $\Gamma=\langle C, N, X, Y, Z$, $u, v, \Lambda\rangle$ satisfy the requirements of Theorem 2.7. Then, the indirect utility function $U^{s}$ is upper semi-continuous.

Proof In Lemma A.1, we make the substitutions, $M=Y, N=X \times Z, D=E$, and $f=U$. We have proven in Lemma A.2 that $E$ is, indeed, upper hemi-continuous as well as non-empty and compact valued. Furthermore, by construction, the utility function $U$ is continuous and, thus, $f=U$ is upper semi-continuous.

Finally, we can see that from Berge's formulation that $V=U^{s}$ by construction. By applying Lemma A.1, we conclude that $U^{s}$ is upper semi-continuous.

We are now in the position to complete the proof of Theorem 2.7.

From Lemma A.3, $U^{s}: Y \rightarrow \mathbb{R}$ is an upper semi-continuous function defined on a compact set $Y$. Hence, it follows from standard results that $\Phi^{s}$ is non-empty.

Take any $y^{*} \in \Phi^{s}$. From Lemma A.2, $E_{y^{*}}$ is non-empty as well as compact. Moreover, $U\left(\cdot, y^{*}, \cdot\right): X \times Z \rightarrow \mathbb{R}$ is a continuous function. Hence, from the Weierstrass Theorem:

$$
\Psi=\underset{(x, z) \in E_{y^{*}}}{\arg \max } U\left(x, y^{*}, z\right) \neq \varnothing .
$$

Take any $\left(x^{*}, z^{*}\right) \in \Psi$. Then, it is easy to establish that $\left(x^{*}, y^{*}, z^{*}\right)$ constitutes a leadership equilibrium in the generalised partial cooperative game $\Gamma$.

This completes the proof of Theorem 2.7.

\section{A.3 Proof of Proposition 3.2}

We proceed by straightforwardly compute the first-order conditions from the optimisation problems for the treaty countries $i \in C$, the independent countries $j \in N$ and for $C$ as a collective. First, notice that without loss of generality, we can replace the inequality in (20) by an equality and re-write the constrained maximisation problem given by Eqs. (16)-(20) as a straightforward maximisation of a single function with two variables given by

$$
\max _{L_{2 h}, d_{h}} u_{h}=\sqrt{\bar{L}-L_{2 h}}+\sqrt{d_{h} L_{2 h}}-\frac{1}{2}\left(\sum_{i=1}^{k} d_{i}+\sum_{j=1}^{n} d_{j}\right) .
$$

The first-order conditions of (31) are given by:

$$
\frac{\partial u_{h}}{\partial L_{2 h}}=-\frac{1}{2 \sqrt{\bar{L}-L_{2 h}}}+\frac{d_{h}}{2 \sqrt{d_{h} L_{2 h}}} \equiv 0
$$




$$
\frac{\partial u_{h}}{\partial d_{h}}=\frac{L_{2 h}}{2 \sqrt{d_{h} L_{2 h}}}-\frac{1}{2} \equiv 0
$$

We re-call that we already established that, for the treaty countries $i \in C$, the equilibrium level of individual country emissions is given by

$$
d_{i}=\frac{L_{2 i}}{k^{2}}
$$

which, through substitution in (32), implies that

$$
\frac{1}{2 \sqrt{\bar{L}-L_{2 i}}}=\frac{1}{2 k} \text {. }
$$

We first show assertion (b) for $\bar{L}>k^{2}$. Hence, $L_{2 i}^{P E}=\bar{L}-k^{2}>0$ and $d_{i}^{P E}=\frac{\bar{L}-k^{2}}{k^{2}}$. In turn, we then establish that $L_{1 i}^{P E}=\bar{L}-L_{2 i}=k^{2}$.

The first-order conditions for the independent countries $j \in N$ now imply the computed outcomes for the Nash equilibrium, i.e., $L_{1 j}^{P E}=1$ and $L_{2 j}^{P E}=d_{j}^{P E}=\bar{L}-1$.

Therefore,

$$
\Delta^{P E}=k\left(\frac{\bar{L}-k^{2}}{k^{2}}\right)+n(\bar{L}-1)=\left(n+\frac{1}{k}\right) \bar{L}-(k+n) .
$$

This implies now that

$$
\begin{aligned}
u_{i}^{P E} & =k+\frac{\bar{L}-k^{2}}{k}-\frac{1}{2}\left(n+\frac{1}{k}\right) \bar{L}+\frac{1}{2}(k+n) \\
& =\frac{1}{2}(k+n)-\frac{1}{2}\left(n-\frac{1}{k}\right) \bar{L} \\
u_{j}^{P E} & =\bar{L}-\frac{1}{2}\left(n+\frac{1}{k}\right) \bar{L}+\frac{1}{2}(k+n) .
\end{aligned}
$$

Now, since $\Delta^{P E}<\Delta^{N E}$, we derive immediately that for every $j \in N: u_{j}^{P E}>u_{j}^{N E}$. Furthermore, for $i \in C$, it holds that $u_{i}^{P E}>u_{i}^{N E}$ if and only if

$$
\frac{\bar{L}-k^{2}}{k}+\frac{1}{2}(3 k+n)-\frac{1}{2}\left(n+\frac{1}{k}\right) \bar{L}>\bar{L}-\frac{1}{2}(k+n) \bar{L}+\frac{1}{2}(k+n)
$$

if and only if

$$
\bar{L}>\left(1-\frac{k}{2}+\frac{1}{2 k}\right) k \bar{L}
$$

Obviously, $\bar{L}>0$, so the inequality simplifies to

$$
\left(1-\frac{k}{2}+\frac{1}{2 k}\right) k<1 \text { or }(k-1)^{2}>0 .
$$


This is obviously the case for any value of $k$ in the assumed range $k \geqslant 2$, showing assertion (b).

Next, we show assertion (a) for $1<\bar{L} \leqslant k^{2}$.

In that case, there is a corner solution for the equilibrium conditions described by $d_{i}^{P E}=L_{2 i}^{P E}=0$ and $L_{1 i}^{P E}=\bar{L}$ for the treaty countries $i \in C$.

For the independent countries $j \in N$, we derive again $L_{1 j}^{P E}=1$ and $L_{2 j}^{P E}=$ $d_{j}^{P E}=\bar{L}-1$.

Thus, $\Delta^{P E}=n(\bar{L}-1)$ and

$$
u_{i}^{P E}=\sqrt{\bar{L}}-\frac{n}{2}(\bar{L}-1) \text { and } u_{j}^{P E}=\bar{L}-\frac{n}{2}(\bar{L}-1)=\left(1-\frac{n}{2}\right) \bar{L}+\frac{n}{2} .
$$

To investigate the Pareto domination between the PE and the NE outcomes, we note again that $u_{j}^{P E}>u_{j}^{N E}$ as $\bar{L}>1$ and now $u_{i}^{P E}>u_{i}^{N E}$ if and only if

$$
\sqrt{\bar{L}}-\frac{n}{2}(\bar{L}-1)>\bar{L}-\frac{1}{2}(k+n)(\bar{L}-1),
$$

if and only if

$$
\sqrt{\bar{L}}>\bar{L}-\frac{k}{2}(\bar{L}-1)
$$

if and only if

$$
2 \sqrt{\bar{L}}>k-(k-2) \bar{L} \text {. }
$$

Now, $\bar{L}>1$ and $k \geqslant 2$ imply that

$$
2 \sqrt{\bar{L}}>2>k-(k-2) \bar{L} .
$$

Hence, $u_{i}^{P E}>u_{i}^{N E}$, thus confirming the assertion of (a) in Proposition 3.2.

\section{References}

Banal-Español, A., Macho-Stadler, I., \& Pérez-Castrillo, D. (2013). Research output from universityindustry collaborative projects. Economic Development Quarterly, 7, 71-81.

Barrett, S. (1990). The problem of global environmental protection. Oxford Review of Economic Policy, 6, $68-79$.

Berge, C. (1997). Topological spaces. Mineola: Dover Publications.

Bernheim, D., \& Whinston, M. D. (1990). Multimarket contact and collusive behavior. RAND Journal of Economics, 21, 1-26.

Billand, P., Bravard, C., Chakrabarti, S., \& Sarangi, S. (2014). A note on networks of collaboration in multimarket oligopolies. Annals of Regional Science, 53, 325-335.

Bloch, F. (1997). Non-cooperative models of coalition formation in games with spillovers. In C. Carraro \& D. Siniscalco (Eds.), New directions in the economic theory of the environment, chapter 10 (pp. 311-352). Cambridge: Cambridge University Press.

Border, K. (1985). Fixed point theorems with applications to economics and game theory. Cambridge: Cambridge University Press. 
Carraro, C., \& Siniscalco, D. (1993). Strategies for the international protection of the environment. Journal of Public Economics, 52, 309-328.

Chakrabarti, S., Gilles, R. P., \& Lazarova, E. (2011). Strategic behaviour under partial cooperation. Theory and Decision, 71(2), 175-193.

Chander, P., \& Tulkens, H. (1997). The core of an economy with multilateral environmental externalities. International Journal of Game Theory, 26, 379-401.

Choi, J. P., \& Gerlach, H. (2013). Multimarket collusion with demand linkages and antitrust enforcement. Journal of Industrial Economics, 61(4), 987-1022.

Finus, M., \& Rundshagen, B. (2009). Membership rules and stability of coalition structures in positive externality games. Social Choice and Welfare, 32, 389-406.

Fudenberg, D., \& Tirole, J. (1991). Game Theory. Cambridge: MIT Press.

Glicksberg, I. L. (1952). A further generalization of the kakutani fixed point theorem. Proceedings of the American Mathematical Society, 3, 170-174.

Helm, D. (2012). Climate policy: The kyoto approach has failed. Nature, 491(7426), 663-665. https://doi. org/10.1038/491663a.

Ichiishi, T. (1981). A social coalitional equilibrium existence lemma. Econometrica, 49, 369-377.

In't Veld, D., \& van Lelyveld, I. (2014). Finding the core: Network structure in interbank markets. Journal of Banking and Finance, 49, 27-40.

Mallozzi, L., \& Tijs, S. (2008a). Conflict and cooperation in symmetric potential games. International Game Theory Review, 10, 245-256.

Mallozzi, L., \& Tijs, S. (2008b). Partial cooperation and multiple non-signatories decision. Czech Economic Review, 2, 23-30.

Mallozzi, L., \& Tijs, S. (2009). Coordinating choice in partial cooperative equilibrium. Economics Bulletin, 29, 1-6.

Mallozzi, L., \& Tijs, S. (2012). Stackelberg vs nash assumption in partially cooperative games. Czech Economic Review, 6, 5-13.

Nash, J. (1950). The bargaining problem. Econometrica, 18, 155-162.

Negishi, T. (1963). On social welfare function. Quarterly Journal of Economics, 77, 156-158.

Newell, R. G., Pizer, W. A., \& Raimi, D. (2013). Carbon markets 15 years after kyoto: Lessons lrearned, new challenges. Journal of Economic Perspectives, 27, 123-146.

Popov, A., \& Ongena, S. (2011). Interbank market integration, loan rates, and firm leverage. Journal of Banking \& Finance, 35, 544-559.

Rawls, J. (1999). A theory of justice. Cambridge: Harvard University Press.

Ray, D. (2007). A game-theoretic perspective on coalition formation. Oxford: Oxford University Press.

Salant, S., Switzer, S., \& Reynolds, R. (1983). Losses from horizontal merger: The effects of an exogenous change in industry structure on cournot-nash equilibrium. The Quarterly Journal of Economics 98 , 185-199. http://www.jstor.org/stable/1885620

White, W. R. (1996). International agreements in the area of banking and finance: Accomplishments and outstanding issues. In G.M. von Furstenberg (Ed.) Regulation and supervision of financial institutions in the NAFTA countries and beyond. Springer, Dordrecht

Yi, S.-S. (1997). Stable coalition structures with externalities. Games and Economic Behavior, 20, 201-237.

Yi, S.-S., \& Shin, H. (2000). Endogenous formation of research coalitions with spillovers. International Journal of Industrial Organization, 18, 229-256. 